\title{
Analysis Of Thumbprint Recognition In Different Bit Levels
}

\author{
M. R. Ramlan \\ Faculty of Engineering \\ University Malaysia Sarawak
}

\author{
D.B.L.Bong \\ Faculty of Engineering \\ University Malaysia Sarawak
}

\author{
T.Z. Lee \\ Faculty of Engineering \\ Universiti Malaysia Sarawak
}

\begin{abstract}
In this paper, a preliminary analysis of thumbprint recognition in different bit levels is presented. A digital image consists of $m \boldsymbol{x} n$ pixels and each pixel is represented by gray level value. In an 8bits gray level image, the gray level value range from 0 to 255 for each pixel. Each bit-plane will then be extracted as the input into the neural network to test its performance and accuracy. The performance is then evaluated based on the recognition rate of each bit plane.
\end{abstract}

Keywords- Thumbprint recognition; bit level extraction; image processing; neural network.

\section{INTRODUCTION}

Biometric technology provides the best solution in security system where human characteristics which is unique for each human is applied. This technology has been used to measure and identify an individual's identity through physiological or behavioral trait [1]. Fingerprint biometric has been the most popular and widely applied among all biometric technologies and has been applied in various applications especially in forensic and access control [2].

The classification of fingerprints was firstly proposed by Galton [3] with three major fingerprint classes that are the arch, whorl and loop. Later, Henry [4] modified Galton's classification and came out with eight classes which consist of Right Loop, Left Loop, Whorl, Plain Arch, Central Pocket, Tented Arch, Accident and Twin Loop. The four major classes of the fingerprint classification are shown in Figure 1.
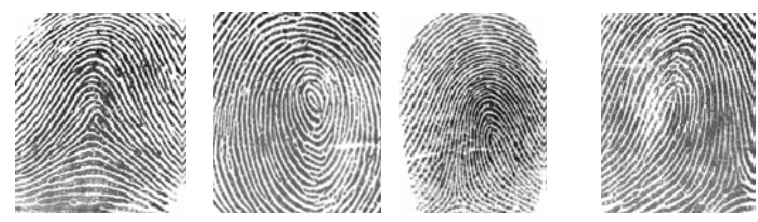

Arch Whorl Right Loop Left Loop
Figure 1. Examples of four major fingerprint classes

There are two major features in a fingerprint/thumbprint that are mostly extracted out for classification: 1) the global ridge and furrow structures located at the central region of the fingerprint which formed special patterns. 2) Local ridge and furrow minute details. Basically, information extraction from ridge structures can be performed by developing a mathematical model of the fingerprint ridge. Also, another method is by recoding the characteristics of the ridges and the information is stored for classification [5].

In fingerprint / thumbprint identification, mostly minutiae-based and correlation-based approaches are implied. For minutiae-based approach, it detects the minutia position, minutia direction, and the type of minutia which is either the ending or bifurcation in a fingerprint / thumbprint image [6][7]. As for correlation-based, it is a process of matching two fingerprint / thumbprint images directly.

Furthermore, gray level and binarized images are usually applied for feature extraction algorithms. Commonly, gray level images are applied with ridge following [8]. As for binarized images, they are used in approaches that involve minutiae detection after the process of thinning the binary image [2]. Some approaches that involve binarization are optimal thresholding, adaptive, and peak detection [9].

Previous research has shown a convincing result in applying bit-plane extraction into face recognition [10]. Hence, in this project, gray level images are used and the thumbprint feature is extracted into different bit levels. Database used here is from FVC2000 [11]. After different bit levels are extracted out from the original image, they are then fed into neural network for classification. Analysis for the thumbprint recognition at different bit level is then performed.

\section{THEORETICAL BACKGROUND}

A digital image is constructed by multilevel information of bits. It consists of $m x n$ pixels and each pixel is represented by gray level value.

As stated by Gonzalez [12], higher order bits consists of most of the information in an image, while lower order bits contains only subtle details. Hence, in an 8-bit image, it will form 8 bit-planes ranging from bit-plane 7 as the highest order bit and bit-plane 0 as the lowest order bit.

Bit level can be extracted mathematically from gray-level image. The gray-level can be derived as in Equation (1).

$f(x, y)=\left[\begin{array}{cccc}f(0,0) & f(0,1) & \ldots & f(0, N-1) \\ f(1,0) & f(1,1) & \ldots & f(1, N-1) \\ \vdots & \vdots & \ldots & \vdots \\ f(M-1,0) & f(M-1,1) & \ldots & f(M-1, N-1)\end{array}\right]$

(1) 